\title{
Modeling of heat transfer in inhomogeneous systems using the flow graph on the example of a lead-acid battery
}

\author{
Mariia Kozlova*, Vitaly Shamansky \\ Melentiev Energy Systems Institute, Siberian Branch of the Russian Academy of Sciences, Lermontov st. 130, Irkutsk, 664033, Russia
}

\begin{abstract}
In this work, it is proposed to represent systems that are heterogeneous in terms of intensive parameters as a set of homogeneous subsystems between which extensive parameters are transferred. With this approach, it is possible to simplify the system by combining subsystems (or already the vertices of a graph) without losing its physical sense. This technique has been applied to simulate a lead acid battery.
\end{abstract}

\section{Introduction}

Dynamical systems are usually described by a set of differential equations. When solving the system of equations numerically, a difference scheme arises, which complicates the program algorithm; moreover, it is not always necessary to calculate the desired parameter at each point of the object under study [1-2]. And one more thing: the addition of a chemical block and possible relief interfaces in the system also greatly complicate the calculations. The representation of the object under study in the form of a graph is a convenient technique for formalizing and solving scientific and engineering problems. In this work, the system under investigation, heterogeneous in intensive parameters (temperature, density, etc.), is described as a set of homogeneous subsystems, between which, according to some physical laws, extensive parameters (heat, mass, etc.) are transferred, which leads to an equilibrium state. Fig. 1 shows the transition from a finite-difference scheme to a graph.

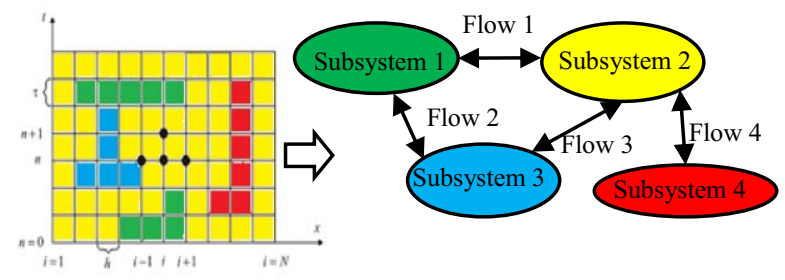

Fig. 1. Graph representation of a finite-difference scheme.

The flows in fig. 1 represent the flows of extensive parameters between subsystems. Each subsystem is homogeneous by intensive parameters.

In this work, this method was used to study the temperature field of the battery during operation. In such a system, the sought-for intensive parameter is the temperature, and between the vertex there pass flows responsible for heat transfer. Recently, electrochemical methods of energy generation and storage have been increasingly used in power engineering. Lead-acid batteries are among the most widely used, along with lithium-ion and nickel-hydrogen batteries: they operate over a wide temperature range $\left(-40^{\circ} \mathrm{C}\right.$ to $\left.+40^{\circ} \mathrm{C}\right)$ and are relatively cheap. However, the optimum operating temperature for this type of battery is around $25^{\circ} \mathrm{C}$. The change in temperature inside the battery is a direct consequence of the thermal effects of the ongoing electrode electrochemical processes that form its energy state. The heat released (or absorbed) as a result of electrochemical reactions is easy to calculate, since it is proportional to the charging (or load) current. The use of this model makes it possible to calculate in detail the thermal modes of operation of storage batteries as part of autonomous power systems. Therefore, it is possible to take into account in detail the own needs of the storage unit depending on the temperature inside the building of the storage room. This formulation and the proposed solution open up the problem of optimizing the battery building, the choice of technical means to ensure the required temperature regime for the operation of the storage batteries. When modeling heat transfer in such structures, it is necessary to know a significant number of parameters [3].

In [3], it is noted that the exact cause of thermalrunaway in lead-acid batteries is unknown despite numerous studies. Numerical results in [3] for VRLAbatteries (in which the products of water electrolysis hydrogen and oxygen molecules - recombine in batteries of this type, turning into water molecules and returning to the electrolyte composition) show that, in addition to Joule heating, only irreversible processes contribute to heat generation, and reversible processes have no contribution. In [3], an attempt was made to identify quantitatively the sources of heat and the cell temperature was found as a function of time. The cell was presented as a set of positive and negative electrodes, a separator between them, a gaseous medium (hydrogen and oxygen) and a surrounding wall. In their work, they used many parameters, such as the porosity

Corresponding author: mari.kozlova.95@mail.ru 
of electrodes and separators, the enthalpies of formation of all substances involved in the reactions, and the linear sizes of the cell components.

An overview of the pros and cons of existing models for lead-acid batteries is given in [4]. In [4], the models are divided by purpose into the following types: models of physical, chemical and electrical phenomena associated with the process of charging / discharging an electrochemical cell; models for predicting external electrical parameters in a stationary charge or discharge mode; electrical equivalent circuits to describe the dynamic behavior of a cell during operation: double layer and diffusion phenomena are replaced by lumped capacitors and resistors.

In [5], models describing the operation of electrochemical energy storage systems are divided into two types: basic resistance models, which can very well model fast dynamics, but are not suitable for slow processes, and physicochemical models, which usually neglect fast dynamics and focus on slow processes. At the same time, the authors note that the distribution of the electric potential and the mass transfer of the electrolyte are important macroscopic effects in the leadacid system. In [6], several parameters related to battery simulation are described: internal resistance, discharge type, discharge mode, charge/discharge rate.

Thus, it is difficult to find one single model of a leadacid battery that would take into account all aspects of its operation. It is also desirable to be able to use the battery manufacturer's data as much as possible in determining the model parameters.

\section{Model}

To create a graph showing the heat exchange inside the battery, a simplified scheme was used, shown in the fig. 2 .

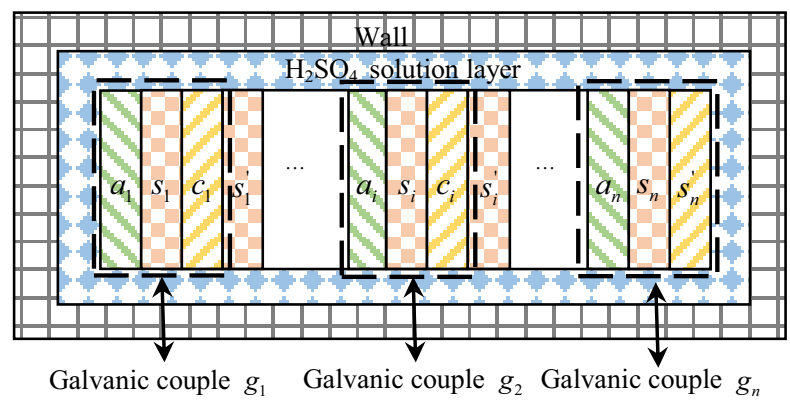

Fig. 2. Battery schematic, where $a_{i}$ - negative electrode (anode); $c_{i}$ - positive electrode (cathode); $s_{i}$ and $s_{i}^{\prime}$ - internal and external separators relative to the galvanic pair, respectively.

The internal sources of heat in the battery are the thermal effects of near-electrode reactions and the heat released during the passage of current through the electrolyte solution and through the current-carrying metal parts of the battery [7]. Since the resistance of metal elements is small, only the first two circumstances can be taken into account in the calculation. It should be taken into account that the passage of current through the electrolyte is limited by the rate of electrochemical reactions. Thus, all thermal effects associated with the absorption or release of heat inside the battery are determined by electrochemical phenomena occurring on the electrodes [3].

The heat exchange inside the battery and with the environment can be represented in the form of an oriented graph, reflecting the connection by heat flows of its constituent galvanic cells (i.e., cathode and anode plates) and with the surrounding sulfuric acid, which borders on the plastic case. The heat flow graph corresponding to the given scheme is shown in the fig. 3 .

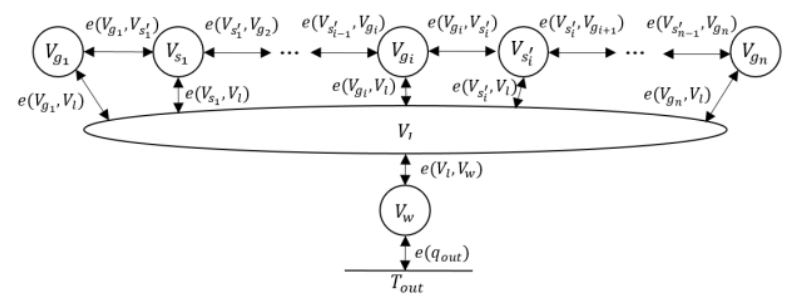

Fig. 3. Battery heat flow graph for block scheme of fig. 2

Arrows at the ends of the arcs indicate the possibility of heat flow in both directions. The graph orientation is established from thermodynamic relations, in this case, depending on the temperature values at the vertices incident to the given arc. The heat flow from the peak with a higher temperature will be considered positive. Such a graph will be called thermodynamically oriented. Since the temperatures of the vertices are determined during the calculation at different times, the orientation of the graph at different stages of the calculations is established in a natural way.

The basis for the formation of a detailed mathematical model will be the balances of heat flows in each vertex of a complex structure in the graph in fig. 3, including the flows (effluent, sources) inside each homogeneous vertex. It should be noted that the presence of internal flows inside any homogeneous vertex appeared when describing the unsteady state of any system with the transfer of extensive parameters, similar to the force of inertia that appears in mechanics when the speed of movement of the elements of a mechanical system changes. The balances (1-7) for the graph will be written as follows:

$$
\begin{gathered}
q\left(V_{g_{1}}, V_{s_{1}^{\prime}}\right)+q\left(V_{g_{1}}, V_{l}\right)+q_{g_{1}}^{V}=0 \\
q\left(V_{g_{1}}, V_{s_{1}^{\prime}}\right)+q\left(V_{s_{1}^{\prime}}, V_{g_{2}}\right)+q_{s_{1}^{\prime}}^{V}=0 \\
q\left(V_{g_{i}}, V_{s_{i-1}^{\prime}}\right)+q\left(V_{g_{i}}, V_{s_{i}^{\prime}}\right)+q\left(V_{g_{i}}, V_{l}\right)+q_{g_{i}}^{V}=0 \\
q\left(V_{g_{i}}, V_{s_{i}^{\prime}}\right)+q\left(V_{s_{i}^{\prime}}, V_{g_{i+1}}\right)+q\left(V_{s_{i}^{\prime}}, V_{l}\right)+q_{s_{i}^{\prime}}^{V}=0 \\
q\left(V_{g_{n}}, V_{s_{n-1}^{\prime}}\right)+q\left(V_{g_{n}}, V_{l}\right)+q_{g_{n}}^{V}=0 \\
\sum_{1}^{n} q\left(V_{g_{i}}, V_{l}\right)+\sum_{1}^{n-1} q\left(V_{s_{i}^{\prime}}, V_{l}\right)+q\left(V_{l}, V_{w}\right)+q_{l}^{V}=0
\end{gathered}
$$




$$
q\left(V_{l}, V_{w}\right)+q_{w}^{V}+q^{\text {out }}=0
$$

In system (1-7), flows are written for the corresponding arcs with the condition that the source vertex is the first in parentheses, and the effluent vertex is the second. Otherwise, the vertices in the stream record should be swapped and a minus sign should be put in front of the stream. The superscript "V" marks the sum of sources (effluent) within the homogeneous peaks that make up the structure. In these relations for one vertex, the sum of all flows incident to it and its internal sinks and sources is equal to zero.

In order to calculate the values of the flows included in the given balances, it is necessary to present in more detail the structure of the vertices of the original graph, since all flows participating in relations (1-7) in the first approximation can be considered additive with respect to their constituent elementary flows ${ }^{\mathrm{a}}$. These flows are then determined on the basis of the known laws of heat transfer and heat transfer. Such a detailed structure of an individual galvanic pair in the form of a graph is shown in fig. 4.

\footnotetext{
${ }^{a}$ In the most general case, of course, it is necessary to take into account the interaction of transporting streams of different nature. As a rule, this happens when the transfer of one component facilitates the transfer of another, for example, convective heat transfer, the phenomenon of thermal diffusion, etc.
}

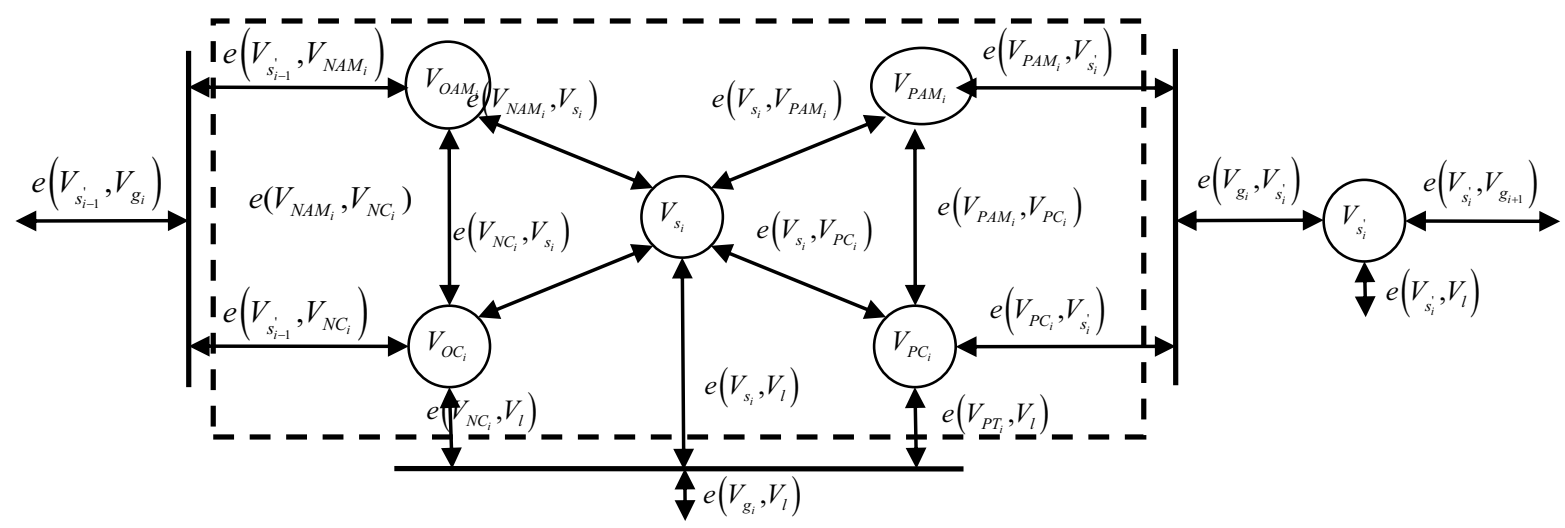

Fig. 4. Simplified flow graph inside a galvanic pair.

The vertices of the graph have a complex structure and are actually subgraphs of the shown general graph. Fig. 4 shows a galvanic pair (anode, separator, cathode) as a set of NAM (negative active mass, that is cells of negative plate), PAM (positive active mass, that is cells of positive plate) and their corresponding down conductors. The construction of the graph is based on the following concepts. Each electrode of a galvanic pair consists of a lead monolithic base, porous components of electrochemical reactions located on it, an electrolyte located in these pores, and also in the pores of a plastic separator separating the electrodes. Heat transfer occurs between these independent phases, as well as heat transfer within them. In our calculations, it is assumed that these phases are temperature homogeneous and there is no heat transfer inside them. In our opinion, this is justified, since the reaction proceeds throughout the entire volume of the porous mass, which is uniformly distributed in the lead grid. In addition, the heat exchange surfaces are relatively large with respect to small heat-conducting distances, which correspond to the thickness of the electrode plates and separators. Note, however, that in cases where the temperature inhomogeneity relative to any direction within the phase cannot be neglected, then a sequence of bivalent vertices is located in this direction, dividing the heat-conducting space into uniform sections. This increases the dimension of the problem, but does not lead to fundamental difficulties in solving it, unless, of course, the dimension of the system becomes too large. Balance ratios for each vertex are constructed how (1-7).

The detail of graph on fig. 3 can be increased, taking into account, for example, the inhomogeneity of the temperature distribution along the perimeter of the electrode plates, the differences in the porous structures of the initial and final products of electrochemical reactions, or the differences between the sulfuric acid solution in the porous structures and the porous structures themselves. However, any detailing of the physical model requires new information about the parameters of the modeled system, the reliability of which often reduces the quality of calculations in comparison with the simplified version. So, to calculate heat flows between a sulfuric acid solution and a porous reaction mass, it is necessary to know the specific surface of the latter and the heat transfer coefficients. It all depends on the values of the temperature gradients between the conditional peaks. If the differences in the temperatures of the incident vertices are small, and the coefficients included in the flow equations are unknown and difficult to determine, it is advisable to combine such vertices into one. In our case, for example, it can be assumed that the heat exchange between the sulfuric acid solution in the pores and the solid phase will proceed intensively due to the large surface, and we can assume that their temperatures are the same ${ }^{\mathrm{b}}$.

\footnotetext{
${ }^{\mathrm{b}}$ It should be noted that the detailing of the graph and its structure primarily depends on the speed parameters of the
} 
The flow balances at the vertices for the graph on fig. 4 are as follows (8-12):

$$
\begin{gathered}
q\left(V_{s_{i-1}^{\prime}}, V_{N A M_{i}}\right)+q\left(V_{N A M_{i}}, V_{s_{i}}\right)+ \\
+q\left(V_{N A M_{i}}, V_{N C_{i}}\right)+q_{N A M_{i}}^{V}=0 \\
q\left(V_{N A M_{i}}, V_{N C_{i}}\right)+q\left(V_{s_{i-1}^{\prime}}, V_{N C_{i}}\right)+q\left(V_{N C_{i}}, V_{s_{i}}\right)+ \\
+q\left(V_{N C_{i}}, V_{l}\right)+q_{N C_{i}}^{V}=0 \\
q\left(V_{N A M_{i}}, V_{s_{i}}\right)+q\left(V_{N C_{i}}, V_{s_{i}}\right)+q\left(V_{s_{i}}, V_{P A M_{i}}\right)+ \\
+q\left(V_{s_{i}}, V_{P C_{i}}\right)+q\left(V_{s_{i}}, V_{l}\right)+q_{s_{i}}^{V}=0 \\
q\left(V_{s_{i}}, V_{P A M_{i}}\right)+q\left(V_{P A M_{i}}, V_{s_{i}^{\prime}}\right)+ \\
+q\left(V_{P A M_{i}}, V_{P C_{i}}\right)+q_{P A M_{i}}^{V}=0 \\
q\left(V_{s_{i}}, V_{P C_{i}}\right)+q\left(V_{P A M_{i}}, V_{P C_{i}}\right)+q\left(V_{P C_{i}}, V_{s_{i}^{\prime}}\right)+ \\
+q\left(V_{P C_{i}}, V_{l}\right)+q_{P C_{i}}^{V}=0
\end{gathered}
$$

where $\mathrm{NC}$ is a negative conductor, $\mathrm{PC}$ is a positive cunductor. This system, taking into account the fact that, as can be seen from the fig. 4 :

$$
\begin{gathered}
q\left(V_{s_{i-1}^{\prime}}, V_{g_{i}}\right)=q\left(V_{s_{i-1}^{\prime}}, V_{O A M_{i}}\right)+q\left(V_{s_{i-1}^{\prime}}, V_{O C_{i}}\right) \\
q\left(V_{g_{i}}, V_{s_{i}^{\prime}}\right)=q\left(V_{P A M_{i}}, V_{s_{i}^{\prime}}\right)+q\left(V_{P C_{i}}, V_{s_{i}^{\prime}}\right) \\
q\left(V_{g_{i}}, V_{l}\right)=q\left(V_{P C_{i}}, V_{l}\right)+q\left(V_{s_{i}^{\prime}}, V_{l}\right)+q\left(V_{P C_{i}}, V_{l}\right) \\
q_{g_{i}}^{V}=q_{N A M_{i}}^{V}+q_{N C_{i}}^{V}+q_{s_{i}}^{V}+q_{P A M_{i}}^{V}+q_{P C_{i}}^{V}
\end{gathered}
$$

allows one to find the temperatures of the homogeneous vertices of the reduced graphs, and, therefore, to calculate the temperature field inside the operating battery and determine the intensity of its heat exchange with the environment. To do this, you need to write down expressions of flows through the parameters of the system based on known patterns. Heat transfer flows at the interface between different phases can be determined based on the Newton law $q_{i, j}^{N}=a_{i, j} S_{i, j}\left(T_{i}-T_{j}\right)$, where $a_{i, j}$ is a heat transfer coefficient; $S_{i, j}$ is an interface area. This kind of flows includes all flows in the balances recorded above, with the exception of flows inside the vertices. The latter should be discussed separately.

phenomena under consideration. So, if the rate of change in the load is comparable to the rates of stages of heterogeneous electrochemical kinetics (for example, the rate of restructuring of the electric double layer or other similar phenomena of molecular order), the detailing of the heat flow graph at this level will be necessary, although it is possible to combine the vertices where heat transfer will much slower, which will allow us to consider areas with slow dynamics as homogeneous (equilibrium).
As mentioned above, each vertex, regardless of its nature, has a "temperature inertia" in the form of a heat absorption law, which for this vertex is written as follows: $Q_{C_{i}}^{V}=\sum_{k} n_{k} C p_{k}^{0}\left(T_{i}-T_{i}^{0}\right)$, where $\mathrm{Q}$ is heat absorbed by the body; $\sum_{k} n_{k} C p_{k}^{0}$ is total heat capacity of a body, heterogeneous in chemical composition, where $n_{k}$ and $C p_{k}^{0}$ are the amount of matter and the heat capacity of the $k^{\text {th }}$ component of the vertex, respectively (for an anode cell: $k=\mathrm{Pb}, \mathrm{H}_{2} \mathrm{SO}_{4}, \mathrm{PbSO}_{4}$, Air ; for a cathode cell: $k=\mathrm{PbO}_{2}, \mathrm{H}_{2} \mathrm{SO}_{4}, \mathrm{PbSO}_{4}$, Air; for a separator: $k=$ Polyethylene, $\mathrm{H}_{2} \mathrm{SO}_{4}$, Air , the material of the separator can be different, as indicated in [8]); $T_{i}^{0}$ and $T_{i}$ are initial and current vertex temperature. Thus, the "inertial" heat flow of the $i^{\text {th }}$ vertex: $q_{i}^{V C}=\sum_{k} n_{k} C p_{k}^{0} \frac{d T_{i}}{d \tau}$. In addition, heat $Q_{r}$ is released at the vertices with electrochemical reactions, which for reversible electrode reactions is equal to $T \Delta S$, where $\Delta S$ is the change in the entropy of the system as a result of chemical transformations. Thus, $q_{r}=\frac{d Q_{r}}{d \tau}=\Delta S \frac{d T}{d \tau}+T \frac{\Delta S}{d \tau}$.

The reactions taking place at the electrodes of a leadacid battery are written as follows (17-18):

$$
\begin{gathered}
(-) \mathrm{Pb}_{2} \mathrm{H}_{2} \mathrm{SO}_{4}-2 \mathrm{e}^{-} \underset{\text { Discharge }}{\square} \mathrm{PbSO}_{4}+2 \mathrm{H}^{+} \\
(+) \mathrm{PbO}_{2}+\mathrm{H}_{2} \mathrm{SO}_{4}+2 \mathrm{H}^{+}+2 \mathrm{e}^{-} \square_{\text {Discharge }}^{\text {Charge }} \mathrm{PbSO}_{4}+2 \mathrm{H}_{2} \mathrm{O}
\end{gathered}
$$

In addition, a concomitant reaction (19-20) of electrochemical decomposition of water occurs on each cathode-anode pair, provided that the potential difference between the plates reaches a hydrogen overvoltage:

$$
\begin{gathered}
(-) 2 \mathrm{H}^{+}+4 \mathrm{e}^{-} \rightarrow 2 \mathrm{H}_{2} \\
(+) 2 \mathrm{H}_{2} \mathrm{O} \rightarrow \mathrm{O}_{2}+4 \mathrm{H}^{+}+4 \mathrm{e}^{-} .
\end{gathered}
$$

It should be noted here that electrode reactions (17)(20) were written for a simplified understanding of the mechanism of these near-electrode chemical transformations. They do not show a multitude of sequential and parallel transient processes with the formation of intermediate components - radicals, basic salts, etc., and the composition of the initial and final products may differ from those given. Nevertheless, since the thermodynamic characteristics of a chemical reaction do not depend on the path of transition between the initial and final states, to calculate the total enthalpy of near-electrode reactions in galvanic battery cells in a first approximation, the balance relations (17)-(20) can be used. In accordance with this, the change in entropy 
$\Delta S_{c}$ at the cathode will be equal to $\Delta S_{2.3}+\Delta S_{2.5}$, and at the anode is $\Delta S_{a}=\Delta S_{2.4}+\Delta S_{2.6}$. The subscripts here correspond to the numbers of the reactions recorded above.

The change in the entropy of the system as a result of the $i^{\text {th }}$ chemical reaction can be written as $\Delta S_{i}=\sum_{j} v_{i j} \Delta n_{i j} S_{j}^{0}=\xi_{i} \sum_{j} v_{i j} S_{j}^{0}=\xi_{i} \Delta S_{i}^{0}$, where $\quad \xi_{i} \quad$ is extent of the reaction; $S_{j}^{0}$ is specific entropy of the $j^{\text {th }}$ substance; $v_{i j}$ is stoichiometric coefficients. Thus it is $q_{r, i}=\Delta S_{i}^{0}\left(\xi_{i} \frac{d T}{d \tau}+T \frac{d \xi_{i}}{d \tau}\right)+\xi_{i} T \frac{d \Delta S_{i}^{0}}{d T} \frac{d T}{d \tau}$. Considering that $\left(\frac{\partial \Delta S_{i}^{0}}{\partial T}\right)_{P}=\frac{\sum v_{i j} C p_{i}^{0}}{T}=\frac{\Delta C p_{i}^{0}}{T}$ and $I=v_{i}^{e} F \frac{d \xi_{i}}{d \tau}$, chemical part is (21):

$$
\begin{aligned}
q_{i}^{V R} & =\Delta S_{i}^{0}\left(\xi_{i} \frac{d T}{d \tau}+T \frac{d \xi_{i}}{d \tau}\right)+\xi_{i} \Delta C p_{i}^{0} \frac{d T}{d \tau}= \\
& =\frac{I \Delta S_{i}^{0}}{v_{i}^{e} F} T+\xi_{i}\left(\Delta S_{i}^{0}+\Delta C p_{i}^{0}\right) \frac{d T}{d \tau} .
\end{aligned}
$$

Current $I$ in (21) is the current flowing through a separate galvanic cell and related to the load current by the ratio $I=I^{l c} / n^{g}$, where $n^{g}$ is the number of parallelconnected pairs in the battery, and the load current meets the condition: $I^{l c} \leq I_{\max }^{l c}$, where $I_{\max }^{l c}$ is load current, the maximum possible at a given rate of electrochemical reactions, more precisely, their limiting stage. Extent of reaction $\xi_{i}$ is the same for cathodic and anodic electrochemical processes: $\xi_{1}$ for (17) and (18) or $\xi_{2}$ for (19) and (20), since these are components of one reaction: $\mathrm{Pb}+\mathrm{PbO}_{2}+2 \mathrm{H}_{2} \mathrm{SO}_{4} \underset{\text { Discharge }}{\stackrel{\text { Charge }}{\rightleftarrows}} 2 \mathrm{PbSO}_{4}+2 \mathrm{H}_{2} \mathrm{O}$ for (17) and (18), $2 \mathrm{H}_{2} \mathrm{O} \rightarrow 2 \mathrm{H}_{2}+\mathrm{O}_{2}$ for (19) and (20). The value $\xi_{1}$ determines the change in the chemical composition of the reaction masses, and therefore characterizes the degree of discharge of the battery cell, considering $\xi_{1}=0$ for the state of a fully charged battery. This value also can be considered proportional to the number of coulombs of electricity received from the battery during its operation.

Since the resistance of the metal conductors in the battery is small, the release of Joule heat will be considered only when current passes through the electrolyte in the separator and in the outer sulfuric acid shell. In this case $q_{i}^{V J}=I^{2} R_{i}=\frac{I^{2}}{\chi_{i}}=\frac{l_{i}}{\chi_{i}^{0} s_{i}^{e l} \beta} I^{2}$ and thus:

$$
q_{l}^{V J}=\frac{l}{\chi^{0} s^{l}} I^{2}
$$

where $\chi^{0}$ is specific conductivity of an aqueous solution of sulfuric acid of a given concentration; 1 is the separator thickness; $s^{e l}$ and $s^{l}$ are the area of separator's surface in contact with the electrode and external solution, respectively; $\beta$ is porosity of plastic base separator. The battery separator allows cells to be designed in such a way as to minimize the space between the respective electrodes while preventing short circuits. To prevent short circuits, separators must act as an insulator when it comes to electronic currents. At the same time, the separator must provide an ion flow between the electrodes so that electrochemical reactions can take place. To achieve both functions, separators are made from non-conductive, acid-resistant porous materials to ensure ionic conductivity: rubber, polyethylene, phenol-formaldehyde resorcinol resin, sintered PVC [8].

Considering the above, the balances for the internal flows of the system are as follows (8-12):

$$
\begin{gathered}
q_{O A M_{i}}^{V}=q_{O A M_{i}}^{V C}+q_{O A M_{i}}^{V R} \\
q_{O C_{i}}^{V}=q_{O C_{i}}^{V C} \\
q_{s_{i}}^{V}=q_{s_{i}^{\prime}}^{V}=q_{s_{i}}^{V C}+q_{s_{i}}^{V J} \\
q_{P A M_{i}}^{V}=q_{P A M_{i}}^{V C}+q_{P A M_{i}}^{V R} \\
q_{P C_{i}}^{V}=q_{P C_{i}}^{V C}
\end{gathered}
$$

Combining balances (1-16) and (25-27), a system of equations is obtained we obtain of the form (28):

$$
(\mathbf{A}+\mathbf{G}) \mathbf{T}+\mathbf{b}=\mathbf{B} \frac{d \mathbf{T}}{d \tau},
$$

where $\mathbf{A}$ is the the Laplacian matrix (or Kirchhoff matrix) defined by the flow graph adjacency matrix, while its elements $A_{i, j}=a_{i, j} S_{i, j}$, where $a_{i, j}$ and $S_{i, j}$ are the heat transfer coefficient and the area of the boundary between the vertex $i$ and the vertex $j$, respectively. This matrix reflects heat transfer according to Newton's law between the vertices. $\mathbf{G}$ is a matrix, taking into account the contribution of internal sources. Vector $\mathbf{B}$ associated with the internal heat capacity of the vertices and their internal effluent and sources. Inhomogeneous part $\mathbf{b}$ also linked to internal sources.

\section{Calculation}

To test the technique, the characteristics of a lead-acid battery $10 \mathrm{OPzS} 1000$ was used, and its dimensions are $72 \times 21 \times 23 \mathrm{sm}$. For a preliminary calculation, it was proposed to consider all electrodes and separators to be the same, combining the same vertices into one vertex, with the exception of the extreme anode and cathode, since their contact area with acid is larger than that of the other electrodes. Thus, it is necessary to solve a system of equations with 11 unknown temperatures: internal NAM, PAM, NC and PC, external NAM, PAM, NC and PC, all separators, a layer of sulfuric acid and a glass wall. The solution was obtained for a equilibrium state when: 


$$
(\mathbf{A}+\mathbf{G}) \mathbf{T}+\mathbf{b}=0
$$

In the calculations, the parameters were used that are given in the tables 1,2 and 3.

Tabel 1. Geometrical parameters.

\begin{tabular}{|c|c|}
\hline Name & Value \\
\hline Number of negatine/positive electrodes & $20 / 20$ \\
\hline Dimensions of negative electrode & $0.54 m \times 0.16 m$ \\
\hline Dimensions of positive electrode & $0.54 m \times 0.16 m$ \\
\hline Dimensions of separator & $0.54 m \times 0.16 m$ \\
\hline Surface proportion of cells & $60 \%$ \\
\hline Porosity Pb (NAM) & $0.57[3]$ \\
\hline Porosity $\mathrm{PbO}_{2}$ (PAM) & $0.53[3]$ \\
\hline Porosity of separator & $0.92[3]$ \\
\hline Saturation of NAM & $0.85 \%[3]$ \\
\hline Saturation PAM & $0.85 \%[3]$ \\
\hline $\mathrm{Saturation}$ od separator $_{3}$ & $0.93 \%[3]$ \\
\hline $\mathrm{H}_{2} \mathrm{SO}_{4}$ concentration & $35 \%$ \\
\hline
\end{tabular}

The heat transfer coefficients presented in table 2 , are indicative.

Tabel 2. Heat transfer coefficients.

\begin{tabular}{|c|c|}
\hline Name & Value \\
\hline Between NAM and NC & $100 \mathrm{~W} /\left(\mathrm{m}^{2} \mathrm{~K}\right)$ \\
\hline Between PAM and PC & $90 \mathrm{~W} /\left(\mathrm{m}^{2} \mathrm{~K}\right)$ \\
\hline Between NAM and separator & $80 \mathrm{~W} /\left(\mathrm{m}^{2} \mathrm{~K}\right)$ \\
\hline Between PAM and separator & $70 \mathrm{~W} /\left(\mathrm{m}^{2} \mathrm{~K}\right)$ \\
\hline Between NC and separator & $120 \mathrm{~W} /\left(\mathrm{m}^{2} \mathrm{~K}\right)$ \\
\hline Between PC and separator & $120 \mathrm{~W} /\left(\mathrm{m}^{2} \mathrm{~K}\right)$ \\
\hline Between NC and $\mathrm{H}_{2} \mathrm{SO}_{4}$ & $150 \mathrm{~W} /\left(\mathrm{m}^{2} \mathrm{~K}\right)$ \\
\hline Between PC and $\mathrm{H}_{2} \mathrm{SO}_{4}$ & $150 \mathrm{~W} /\left(\mathrm{m}^{2} \mathrm{~K}\right)$ \\
\hline Between separator and $\mathrm{H}_{2} \mathrm{SO}_{4}$ & $60 \mathrm{~W} /\left(\mathrm{m}^{2} \mathrm{~K}\right)$ \\
\hline Between NAM and $\mathrm{H}_{2} \mathrm{SO}_{4}$ & $20 \mathrm{~W} /\left(\mathrm{m}^{2} \mathrm{~K}\right)$ \\
\hline Between PAM and $\mathrm{H}_{2} \mathrm{SO}_{4}$ & $19 \mathrm{~W} /\left(\mathrm{m}^{2} \mathrm{~K}\right)$ \\
\hline Between $\mathrm{H}_{2} \mathrm{SO}_{4}$ and glass wall & $100 \mathrm{~W} /\left(\mathrm{m}^{2} \mathrm{~K}\right)$ \\
\hline Between glass wall and environment & $150 \mathrm{~W} /\left(\mathrm{m}^{2} \mathrm{~K}\right)$ \\
\hline
\end{tabular}

Table 3 shows the thermophysical parameters.

Tabel 3. Thermophysical parameters [9].

\begin{tabular}{|c|c|}
\hline Name & Value \\
\hline $\mathrm{PbO}_{2}$ heat capacity & $65 \mathrm{~J} /(\mathrm{mole} \cdot \mathrm{K})$ \\
\hline $\mathrm{PbO}_{2}$ density & $9330 \mathrm{~kg} / \mathrm{m}^{3}$ \\
\hline $\mathrm{Pb}$ heat capacity & $140 \mathrm{~J} /(\mathrm{kg} \cdot \mathrm{K})$ \\
\hline $\mathrm{Pb}$ density $_{2}$ & $11340 \mathrm{~kg} / \mathrm{m}^{3}$ \\
\hline $\mathrm{H}_{2} \mathrm{SO}_{4}$ heat capacity & $3000 \mathrm{~J} / \mathrm{kg} \cdot \mathrm{K})$ \\
\hline $\mathrm{H}_{2} \mathrm{SO}_{4}$ density & $1300 \mathrm{~kg} / \mathrm{m}^{3}$ \\
\hline $\mathrm{PbSO}_{4}$ heat capacity & $103 \mathrm{~J} /(\mathrm{mole} \cdot \mathrm{K})$ \\
\hline $\mathrm{PbSO}_{4}$ density & $6350 \mathrm{~kg} / \mathrm{m}^{3}$ \\
\hline Separator's base heat capacity & $1780 \mathrm{~J} /(\mathrm{kg} \cdot \mathrm{K})$ \\
\hline Separator's base density & $950 \mathrm{~kg} / \mathrm{m}^{3}$ \\
\hline $\mathrm{Glass}^{3}$ wall heat capacity & $500 \mathrm{~J} /(\mathrm{kg} \cdot \mathrm{K})$ \\
\hline Glass wall density & $3000 \mathrm{~kg} / \mathrm{m}^{3}$ \\
\hline Air heat capacity & $1030 \mathrm{~J} /(\mathrm{kg} \cdot \mathrm{K})$ \\
\hline Air density & $1,29 \mathrm{~kg} / \mathrm{m}^{3}$ \\
\hline $\mathrm{H}_{2} \mathrm{SO}_{4}$ conductivity & $73 \mathrm{om}-1 \cdot \mathrm{m}-1$ \\
\hline Separator's conductivity & $0 \mathrm{om}-1 \cdot \mathrm{m}-1$ \\
\hline
\end{tabular}

Fig. 5 shows the calculations of the temperature field taking into account the extreme electrodes. Temperature values have been rounded to the nearest hundredth.

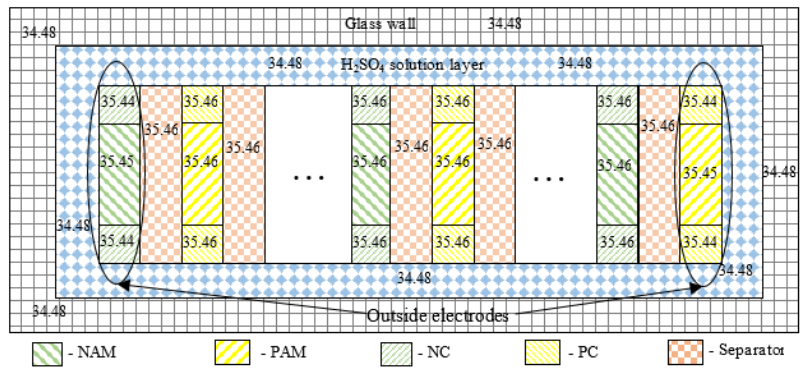

Fig. 5. Temperature distribution in the lead-acid battery at $T=27^{\circ} \mathrm{C}, I=30 \mathrm{~A}$ considering Joule heat.

As can be seen from fig. 5, the temperatures of the outermost electrodes are lower than the internal temperatures, which is logical, since these electrodes are cooled faster due to more intense heat exchange with the surrounding sulfuric acid.

Fig. 6 shows a comparison of element temperatures depending on load, operating mode and Joule heat accounting.
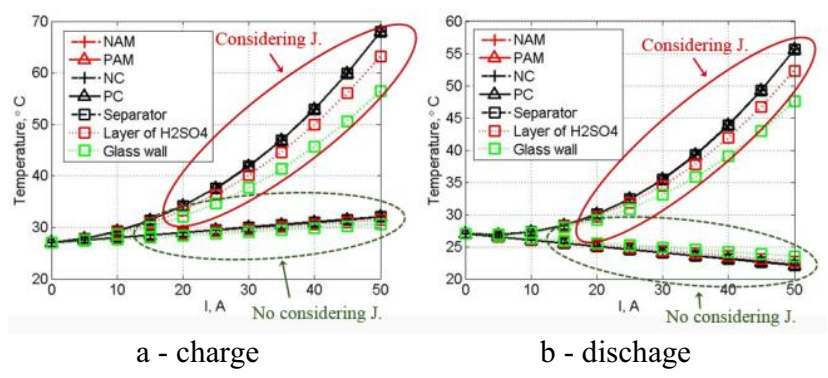

Fig. 6. The temperature of the elements inside the battery, depending on the Joule heat accounting: a - charge, b discharge. There is the Joule heat taken into account in the solid oval, there is without Jole heat accounting in the dashed one.

As can be seen from fig. 6 the main source of heat is Joule heating in separators, and the charge and discharge reactions are compensating for each other, which corresponds to the second concept in [3], according to which only Joule heat affects heating, for example, of a VRLA battery. For non-VRLA batteries, the hydrogen and oxygen formed as a result of electrolysis do not return to the composition of water, and then one should expect a contribution not only from Joule heating, but also from the irreversible part of the processes in the battery (electrolysis).

\section{Conclusion}

To describe a system with a complex, heterogeneous and multicomponent structure, it is important to develop new approaches that simplify a computational experiment by changing the principles of constructing a mathematical model. The transition from a representation in the form of a difference scheme to a flow graph makes it possible to simulate the dynamics of systems of varying complexity.

The studied non-equilibrium system is split into local-equilibrium subsystems connected with each other by the flows of extensive parameters: for example, in 
this work, the vertices of the battery are connected by heat exchange according to a linear law. The vertices of such a graph are the points located at the nodes of the difference scheme, and the arcs are the connections between these points. The weights of the vertices and arcs of the graph are calculated based on the properties of the modeled system. The resulting graph corresponds to a system of differential equations with respect to the vertices, which in these circumstances correspond to the coordinates of the phase space of the given system, and the number of vertices is equal to the dimension of this space. This system shows the change in the state of the object over time. The number of vertices and edges and their weight can change during the computation, which creates the dynamics of the graph, and then, the so-called dynamic graphs will be required to describe such a system.

Such a flow graph representation is, in fact, Lagrangian, since coordinates' derivatives are not in the system of differential equations. This simplifies the formalization and solution of the problem of finding the distribution of certain parameters.

In this work, the presented method was applied to a lead-acid battery model $10 \mathrm{OPzS} 1000$. The solution was obtained for a equilibrium state. In the calculations performed, it was shown that the Joule heat makes the greatest contribution to the temperature rise of this type of batteries, which does not contradict the known data.

Using the proposed approach, it is possible to adjust the model in accordance with other aspects of battery's operation: taking into account the maximum possible load; analysis of transient processes; electrolysis of water; and etc.

This work is performed in the course of scientific project III.17.1.2 of Siberian Branch of Russian Academy of Sciences fundamental researches program, registration number AAAAA17-117030310448-0

\section{References}

1. M. N. S. Swam, K. Thulasiraman, Graphs, Networks, and Algorithms (1980).

2. A.N. Kurganskyy, A.J. Maksimova Trudi IPMM, 30, 96-108 (2018).

3. F. Torabi, V. Esfahanian, J. Electrochem. Soc, 160 (2012).

4. D. Baert, A. Vervaet, Electrochim. Acta, 44, 34913504 (1999).

5. M. Huck, D. U. Sauer, J. Energy Storage, 29 (2020).

6. J. F. Manwell, J. G. Mcgowan, 50, 5, 399-405 (1993).

7. U. B. Kamenev, A. V. Kiselevich, V. N. Leonov, Elecrtochem. Energy, 8, 140-145 (2008).

8. J. K. Whear, Lead-Acid Battery Technologies: Fundamentals, Materials, and Applications (2016).

9. V. V. Batrakov, V. P. Batrakov, Korrozia konstrukcionnih materialov (Metallurgia, 1990). 